\title{
Ocean Currents-Induced Pipeline Lateral Stability on Sandy Seabed
}

\author{
Fu-Ping Gao'; Shuming Yan²; Bing Yang ${ }^{3}$; and Yingxiang $\mathrm{Wu}^{4}$
}

\begin{abstract}
Unlike previous mechanical actuator loading methods, in this study, a hydrodynamic loading method was employed in a flow flume for simulating ocean currents induced submarine pipeline stability on a sandy seabed. It has been observed that, in the process of pipeline losing lateral stability in currents, there usually exist three characteristic times: (1) onset of sand scour; (2) slight lateral displacement of pipeline; and (3) breakout of pipeline. An empirical linear relationship is established between the dimensionless submerged weight of pipeline and Froude number for describing pipeline lateral stability in currents, in which the current-pipe-soil coupling effects are reflected. Scale effects are examined with the method of "modeling of models," and the sand particle size effects on pipeline stability are also discussed. Moreover, the pipeline stability in currents is compared with that in waves, which indicates that the pipeline laid directly upon the sandy seabed is more laterally stable in currents than in waves.
\end{abstract}

DOI: 10.1061/(ASCE)0733-9399(2007)133:10(1086)

CE Database subject headings: Submarine pipelines; Ocean waves; Stability; Sea floor; Hydrodynamics.

\section{Introduction}

One of main problems encountered with the use of submarine pipeline for transport of oil and gas is the pipeline stability on the seafloor under ocean environmental loads. When a submarine pipeline is installed upon the seabed, there exists a complex interaction between pipeline, soil, and hydrodynamic loads. To keep the pipeline stable on the seabed, the soil must provide enough resistance to balance the hydrodynamic loads. Otherwise, the pipeline may break out from its original site, i.e., the pipeline loses on-bottom stability.

In the recommended practice for on-bottom stability design of submarine pipeline (Det Norske Veritas 1988), the weight of concrete coating is a decisive factor for the accomplishment of satisfactory pipeline stability, and it may prove to be the decisive factor for carrying out the installation. To avoid the occurrence of lateral instability of pipelines, the thickness of the concrete coating usually has to be increased. Nevertheless, a slight increase of the coating thickness would bring a larger laying cost, and sometimes might cause the existing laying barge to become unworkable (Allen et al. 1989). Alternatively, the pipeline has to be

\footnotetext{
${ }^{1}$ Associate Professor, Institute of Mechanics, Chinese Academy of Sciences, Beijing 100080, China (corresponding author). E-mail: fpgao@imech.ac.cn

${ }^{2} \mathrm{Ph} . \mathrm{D}$. Student, Institute of Mechanics, Chinese Academy of Sciences, Beijing 100080, China.

${ }^{3}$ Ph.D. Student, Institute of Mechanics, Chinese Academy of Sciences, Beijing 100080, China.

${ }^{4}$ Professor, Institute of Mechanics, Chinese Academy of Sciences, Beijing 100080, China.

Note. Associate Editor: Nikolaos D. Katopodes. Discussion open until March 1, 2008. Separate discussions must be submitted for individual papers. To extend the closing date by one month, a written request must be filed with the ASCE Managing Editor. The manuscript for this paper was submitted for review and possible publication on May 16, 2006; approved on March 14, 2007. This paper is part of the Journal of Engineering Mechanics, Vol. 133, No. 10, October 1, 2007. CASCE, ISSN 0733-9399/2007/10-1086-1092/\$25.00.
}

anchored or trenched, which would increase economic investment and also complicate the design and construction. In the current pipeline engineering practice, mistakes or inconsistencies often occur in the design of pipeline (Lawlor and Flynn 1991). Therefore, an appropriate criterion for pipeline lateral stability is greatly needed for determination of the pipeline's submerged weight.

In the past few decades, the dynamics interactions between wave/current-pipeline-seabed have attracted much interest from pipeline researchers and designers. In the 1980s, the pipeline lateral stability has been investigated mainly by physical modeling in a few large projects, such as the PIPESTAB project (Wagner et al. 1987), the AGA project (Brennodden et al. 1989), and a project at DHI (Palmer et al. 1988). On the basis of their test data, a few pipe-soil interaction models have been proposed for predicting pipeline stability on sand or on clay (e.g., Wagner et al. 1987; Brenodden et al. 1989). Recently, a series of centrifuge tests were conducted on the pipe-soil interaction for a shallowly embedded pipeline in calcareous sand. A nonassociated bounding surface model was also constructed to simulate the soil responses around the pipeline under combined vertical and horizontal monotonic loading (Zhang et al. 2002). In the above experimental investigations, the mechanical actuators were employed in the tests for the simulation of hydrodynamic wave loads upon pipe sections. Thus, only the wave forces upon the pipe were taken into account. However, the wave loads upon the seabed were ignored in those pipe-soil interaction tests. It should be noted that, in the actual situations, the waves or currents not only exert loads upon the pipeline, but also upon the seabed. That is, the pipeline lateral stability in ocean environments is essentially the dynamic interaction between wave/current, pipe and seabed.

In contrast to the previously mentioned mechanical actuator loading methods, a hydrodynamic loading method with a unique U-shaped oscillatory flow tunnel was adopted by Gao et al. (2002), for physical modeling of wave-induced pipeline lateral stability. Based on the wave-pipe-soil interaction model (Gao et al. 2002, 2003), an improved analysis method for waveinduced pipeline lateral stability on the sandy seabed was recently 
proposed by Gao et al. (2006). In addition, the wave-induced stability of submarine pipelines on a mobile seabed was further investigated experimentally in a wave flume by Teh et al. (2003). However, in most of the aforementioned studies, the ocean wave is the main concern of environmental loads.

At certain ocean zones in shallow waters and most locations in deep waters, ocean currents may be the dominant environmental loads. Until now, however, the work specifically focusing on the currents induced pipeline lateral stability, such as the work by Jones (1978), is still quite scarce. The existing experiments regarding current-induced pipeline lateral stability were conducted with mechanical actuators in monotonic loading conditions (e.g., Brennodden et al. 1986; Palmer et al. 1988). Although the pipe on-bottom stability in currents sounds less complex than in waves, until now the underlying physical mechanism has not been revealed. An appropriate model for accurately estimating pipeline stability in the currents is highly desired, especially when the operation of oil and gas exploitation is currently moving into deeper waters.

In this paper, similarity analysis is made on ocean currents induced pipeline stability on the sandy seabed under the influence of ocean currents. According to the established similarity relationships, a series of tests have been conducted by means of the hydrodynamic loading method. The process of pipeline losing stability in currents was recorded and analyzed. Based on the experimental results, an empirical formula will be established for predicting the currents induced lateral instability of freely laid pipelines on sands. Furthermore, a comparison is made between the pipeline lateral stability in currents and that in waves.

\section{Similarity Analysis}

The lateral stability of a pipeline under the influence of ocean currents is a dynamic interaction between pipeline, soil, and flow. To properly describe the pipeline dynamic responses, it is not easy to formulate analytical expressions. Nevertheless, the similarity analysis method can be utilized for analyzing these kinds of problems (Chakrabarti 1994). With the similarity analysis method, the predominant dimensionless parameters can be derived from the parameters involved.

The main influential factors in the aforementioned coupling problem include the characteristic parameters of ocean currents, pipeline, and sandy seabed, as listed in Table 1. The critical pipeline submerged weight $\left(W_{s}\right)$ to keep the pipeline stable in a steady flow is mainly relative to the following parameters

$$
W_{s}=f\left(D, U, t, \nu, \rho_{\mathrm{sat}}, \rho_{w}, g, d_{s}, D_{r}, \kappa\right)
$$

The explanations of the symbols in Eq. (1) are given in Table 1.

Based on the Vaschy-Buckinghan's theorem, the independent dimensionless parameters may be obtained. A functional relationship that represents the currents induced pipeline instability on sand may be expressed as

$$
G=f^{\prime}\left(\mathrm{F}, \mathrm{R}, \frac{t U}{D}, \frac{\rho_{\mathrm{sat}}}{\rho_{w}}, \frac{D}{d_{s}}, D_{r}, \kappa\right)
$$

where

$$
G=\frac{W_{s}}{\gamma^{\prime} D^{2}}
$$

$=$ nondimensional submerged weight of the pipeline; and $\gamma^{\prime}=\left(\rho_{\text {sat }}-\rho_{w}\right) g=$ buoyant unit weight of sands
Table 1. Main Parameters for Modeling Ocean Currents-Induced Pipeline Instability on Sandy Seabed

\begin{tabular}{lcc}
\hline Parameter & Symbol & Dimension \\
\hline Properties of ocean currents & & \\
Flow velocity & $U$ & $\mathrm{~L} \mathrm{~T}^{-1}$ \\
Loading time & $t$ & $\mathrm{~T}$ \\
Kinematic viscosity of water & $v$ & $\mathrm{~L}_{2} \mathrm{~T}^{-1}$ \\
Properties of pipe & $D$ & \\
Pipe diameter & $W_{s}$ & $\mathrm{~L} \mathrm{~T}^{-2}$ \\
Submerge weight of pipe per meter & $\kappa$ & 1 \\
Relative roughness of pipe surface & & \\
Properties of sandy seabed & $\rho_{\mathrm{sat}}$ & $\mathrm{M} \mathrm{T}^{-3}$ \\
Density of saturated sand & $\rho_{w}$ & $\mathrm{M} \mathrm{T}^{-3}$ \\
Density of pore water & $g$ & $\mathrm{~L} \mathrm{~T}^{-2}$ \\
Gravitational acceleration & $d_{s}$ & $\mathrm{~L}$ \\
Sand diameter & $D_{r}$ & 1 \\
Relative density of sand & &
\end{tabular}

$$
\mathrm{F}=\frac{U}{\sqrt{g D}}
$$

$=$ Froude number, which is the ratio of inertia force to gravitational force and reflects the dynamic similarity of flow with gravity forces acting

$$
\mathrm{R}=\frac{U D}{v}
$$

$=$ Reynolds number, which is the ratio of inertia force to viscous force; $t U / D=$ nondimensional loading time of ocean current; $\rho_{\text {sat }} / \rho_{w}=$ specific gravity of saturated sand (the ratio of the density of saturated sand to that of pore water); $D / d_{s}=$ ratio of the pipe outer diameter to the sand diameter; and the explanation of $D_{r}$ and $\kappa$ are given in Table 1 .

From the above similarity analysis, two dimensionless parameters relative to flow characteristics, i.e., Froude number $(F)$ and Reynolds number $(R)$, are deduced for the wave-pipeline-soil interaction problem. In the case of ocean currents with a free surface, the gravitational effect predominates. Moreover, the submerged weight of the pipeline also has a significant influence on the pipeline lateral stability. The effects of other factors, such as viscosity, surface tension of water, etc., are generally small and ignorable. The $\mathrm{F}$ range in the experiments is from 0.1 to 0.4 , which is the representative value in the fields. The $R$ range in the experiments (on the order of $10^{3}-10^{4}$ ) is approximately one order less than in the prototypes (on the order of $10^{5}$ ). As both $F$ and $R$ cannot be satisfied concurrently in the scaled model tests, it is convenient to employ the Froude scaling process and allow the variation of Reynolds number to a certain degree (Chakrabarti, 1994).

According to principle of similarity, the following scales for Froude parameter should be maintained

$$
\frac{\lambda_{U}}{\lambda_{g}^{1 / 2} \lambda_{D}^{1 / 2}}=1
$$

in which $\lambda$ represents the ratio of the parameter of model to that of prototype. As $\lambda_{g}=1$, thus 
Table 2. Similarity Relationships for Currents-Induced Pipeline Stability

\begin{tabular}{lccc}
\hline Parameter & Criterion & Similarity & Ratio \\
\hline$D$ & & $\lambda_{D}$ & $1 / n$ \\
$\gamma^{\prime}$ & & $\lambda_{\gamma^{\prime}}$ & 1 \\
$W_{s}$ & $G$ & $\lambda_{W_{s}}=\lambda_{\gamma^{\prime}} \lambda_{D}^{2}$ & $1 / n^{2}$ \\
$U$ & $\mathrm{~F}$ & $\lambda_{U}=\lambda_{g}^{1 / 2} \lambda_{D}^{1 / 2}$ & $1 / \sqrt{n}$ \\
$t$ & $U t / D$ & $\lambda_{t}=\lambda_{D} / \lambda_{U}$ & $1 / \sqrt{n}$ \\
\hline
\end{tabular}

$$
\begin{gathered}
\lambda_{U}=\lambda_{D}^{1 / 2} \\
\lambda_{t}=\lambda_{D} / \lambda_{U}=\lambda_{D}^{1 / 2}
\end{gathered}
$$

In our experiments, $\rho_{\text {sat }} / \rho_{w}$ remains constant for the series of tests on the same sand, and the pore media and liquid in flume are water (i.e., $\lambda_{\rho_{w}}=1$ ), thus $\lambda_{\gamma^{\prime}}=1$. To maintain the similarity of nondimensionless parameter $G, \lambda_{W_{s}}$ should be equal to $\lambda_{D}^{2}$. The main similarity relationships of the above dimensionless parameters are listed in Table 2, in which $n=$ scale factor, i.e., the dimension ratio between prototype and model.

\section{Experimental Setup and Testing Method}

The tests were conducted in a water flow channel (length $\times$ width $\times$ height $=19.0 \times 0.5 \times 0.6 \mathrm{~m}^{3}$ ), which is capable of generating steady currents with velocity up to approximately $0.4 \mathrm{~m} / \mathrm{s}$. The test pipe was laid directly upon a model seabed and the current was flowing perpendicularly to the pipe axis for two-dimensional simulation of a long distance laid pipeline, as illustrated in Fig. 1.

Three diameters of model pipes with various submerged weights were used, i.e., $D=0.050,0.040$, and $0.032 \mathrm{~m}$ respectively. Note that only smooth pipes $(\kappa \approx 0)$ are considered in this study. The length of test pipe is $0.47 \mathrm{~m}$ and the water depth is $0.4 \mathrm{~m}$. For simulating increasing continuous ocean currents, a typical current with flow acceleration of approximately $1.7 \times 10^{-4} \mathrm{~m} / \mathrm{s}^{2}$ was adopted. The flow velocity was measured with a minikinemometer located in the front of test pipe and above the seabed. A slice light was generated for the purpose of vortex and sand scour observation. The vortex shedding from the test pipe was visualized by the flow field visualization technique with hydrogen bubbles. Meanwhile, the pipe displacement, sand scouring, and the vortex that formed around the test pipe were recorded with a digital video camera (see Fig. 1).

Two kinds of sands were used in the experiments, i.e., medium and fine, whose particle distribution is given in Fig. 2. The index

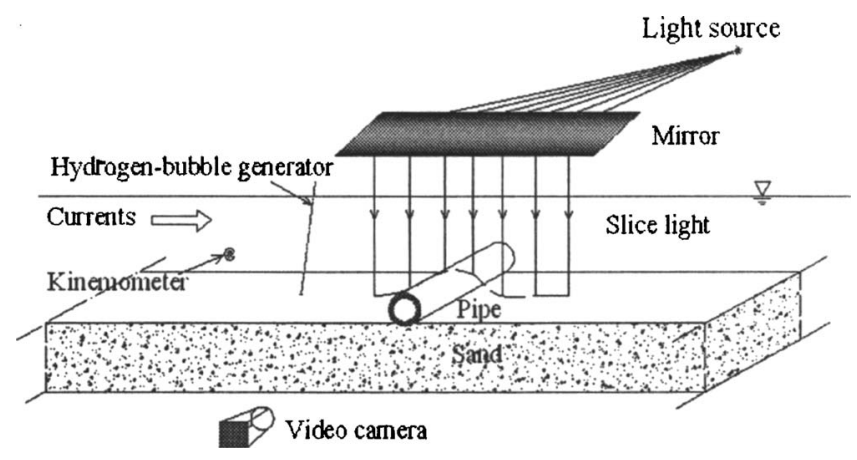

Fig. 1. Schematic diagram of experimental setup

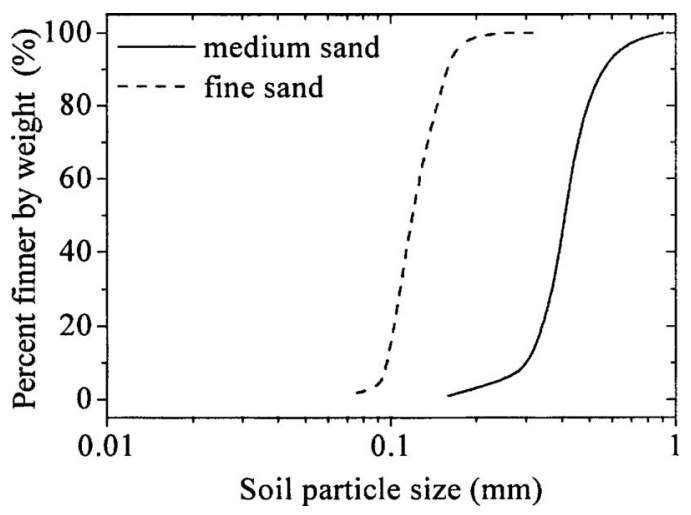

Fig. 2. Grain size distribution curves of two kinds of sandy seabed

properties of the two kinds of test sands are listed in Table 3, where $d_{50}=$ mean grain size of soil; $d_{10}=$ grain size at which $10 \%$ of the soil weight is finer (also called efficient diameter); $C_{u}=$ uniformity coefficient of soil; and $e_{0}=$ initial void ratio of soil. As shown in the table, the two kinds of sands are both of medium-dense sands $\left(1 / 3<D_{r}<2 / 3\right)$.

\section{Experimental Results and Discussion}

\section{Characteristic Times during Pipe Losing Lateral Stability in Currents}

The physical process of the pipeline losing lateral stability was simulated in a flume for a series of test pipes with various submerged weight on the two kinds of sandy soil. When a submarine pipeline is directly laid upon a sandy seabed, there always exists an initial embedment [see Fig. 3(a)]. Under the influence of an increasing ocean current loading, the pipeline may break out from its original site, i.e., pipeline lateral instability occurs, as shown in Fig. 3(b). A typical process of pipeline losing lateral stability in increasing continuous ocean currents is illustrated in Fig. 4.

In accordance with the experimental observations, it can be summarized that there always exist three characteristic times in the process of the pipeline losing lateral stability, as shown in Fig. 5:

1. $t=t_{s}$ : onset of sand scour around pipe. When the flow velocity is increased to a certain value, the sand scour around the pipeline will occur. The flow visualization shows that the onset of sand scour results from the intensive interaction of the vortex with soil, which is located behind the test pipe with a distance of about 3-5 times the pipe diameter. By increasing the flow velocity, the sand scour zone is enlarged. In the process of local scouring, the sands in front of the pipe are moving towards the pipe, and the sands behind pipe are being scoured away from pipe.

2. $t=t_{r}$ : slight lateral displacement of pipe. At a certain flow velocity, there exists a slight lateral displacement of pipe. That is, the pipe pushes the soil with a perceptible horizontal

Table 3. Index Properties of Two Kinds of Test Sands

\begin{tabular}{lcccccc}
\hline & $\begin{array}{c}d_{50} \\
(\mathrm{~mm})\end{array}$ & $\begin{array}{c}d_{10} \\
(\mathrm{~mm})\end{array}$ & $C_{u}$ & $\begin{array}{c}\gamma^{\prime} \\
\left(\mathrm{kN} / \mathrm{m}^{3}\right)\end{array}$ & $e_{0}$ & $D_{r}$ \\
\hline Medium sand & 0.38 & 0.30 & 1.46 & 9.3 & 0.73 & 0.4 \\
Fine sand & 0.12 & 0.09 & 1.41 & 8.7 & 0.86 & 0.6 \\
\hline
\end{tabular}




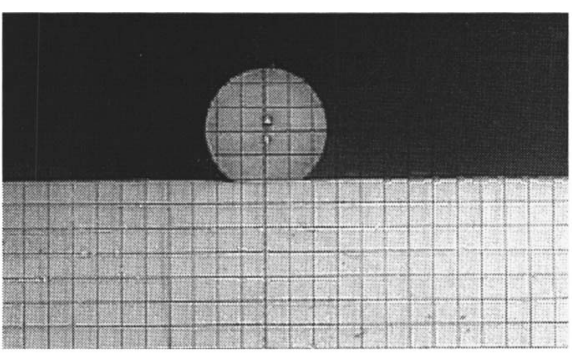

(a)

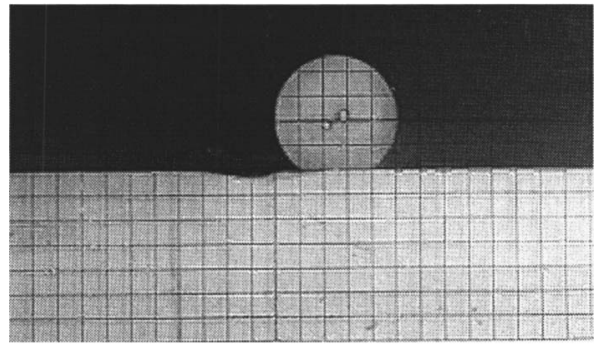

(b)

Fig. 3. Pipeline losing lateral stability in ocean currents: (a) initial embedment of pipe; (b) pipe breakout from original site

displacement, which is also accompanied by pipe rolling. Then the pipe remains still for a period of time (e.g., a few minutes, see Figs. 4 and 5). In this and the later phases, local scouring around the pipe is still being involved.

3. $t=t_{b}$ : pipe breakouts. When the flow velocity becomes high enough, the pipe will break out suddenly from its original site with a large lateral displacement (see Figs. 4 and 5). The duration of this phase, i.e., from "the slight lateral displacement of pipe" to "the breakout of pipe," lasts even longer for the higher values of submerged weights of the pipe.

\section{Correlation of Froude Number with Dimensionless Submerged Weight of Pipeline}

One of the main purposes of this study is to investigate the influence of the submerged weight of the pipeline on its lateral stability in ocean currents. To this aim, 27 series of tests have

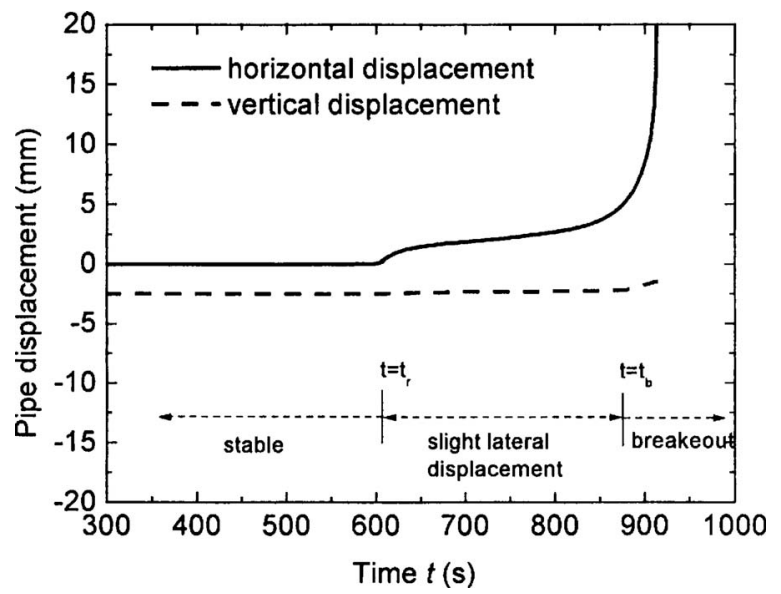

Fig. 4. Pipe displacement-time curves in process of losing lateral stability in currents

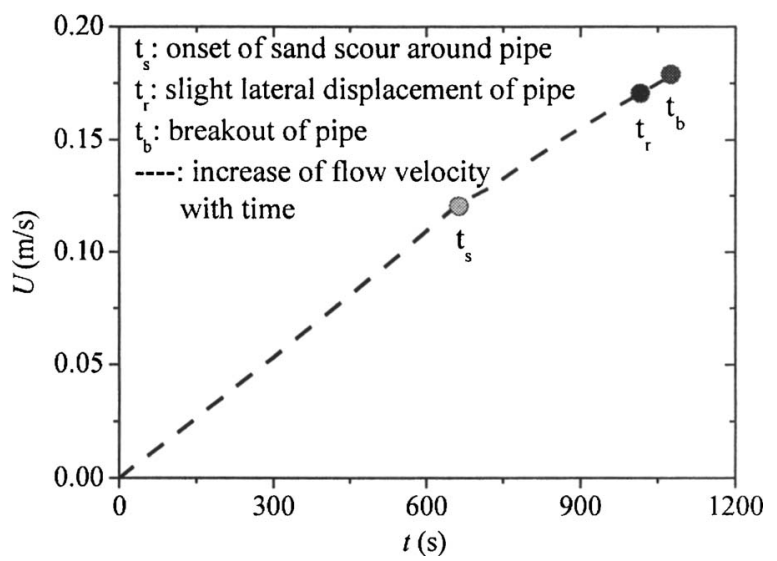

Fig. 5. Typical process of pipeline losing lateral stability on sandy seabed $\left(D=0.050 \mathrm{~m}, W_{s}=7.096 \mathrm{~N} / \mathrm{m}\right.$, medium sand $)$

been conducted on a medium-sand seabed, whose main properties are given in Table 2 in the above section. As previously mentioned, once the submerged weights of the pipeline are determined, the thickness of the concrete cover of pipeline can thereby be given in the practice of pipeline lateral stability design. Therefore, a suitable correlation between the pipeline submerged weight and the dominant environmental parameters is essential for the proper stability design of a submarine pipeline.

In our experiments, three diameters of pipes, i.e., $D=0.050$, 0.040 , and $0.032 \mathrm{~m}$, with various submerged weights, have been tested. In each experiment, for a given value of submerged weight of pipe $\left(W_{s}\right)$, the velocity of currents inducing pipe lateral stability $(U)$ was measured with a minikinemometer (see Fig. 1). The dimensionless parameter $\mathrm{F}$ and $G$ can thereby be calculated according to Eqs. (3) and (4). The experimental results of ocean currents induced lateral stability of pipeline on the medium sand are listed in Table 4. The F- $G$ correlation for the lateral stability of pipes on a medium sand is obtained (see Fig. 6). As shown in Fig. 6, there exists a linear relationship between Froude number (F) and the dimensionless pipeline submerged weight $(G)$ for describing the lateral stability on sand within the examined Froude range $(0.10<\mathrm{F}<0.35)$

$$
\mathrm{F}=0.102+0.423 G \quad \text { (for a medium-sand seabed) }
$$

In the above empirical relationship for describing ocean currents induced pipeline instability (or called the current-pipe-soil interaction model), the main parameters of flow, pipeline, and soil are included. The coupling between flow, pipe, and soil is reflected in the empirical model, which provides a better insight into the physical mechanism for the pipeline lateral instability in currents than the pipe-soil interaction models proposed by Wagner et al. (1987).

\section{Discussion on Scale Effects}

In physical modeling studies, it is seldom possible to replicate precisely all details of the prototype and some approximations have to be made. Although the model may not be an exact replica of the prototype, it is still a unique physical event. It is important to recognize that model studies are not perfect and to inquire into the nature of any shortcomings, often referred to as scale effects. A good technique for checking for scale effects is the "modeling of the models," which is particularly useful when no prototype is available for verifying the model (Taylor 1995). 
Table 4. Experimental Results of Currents-Induced Lateral Stability of Pipeline on Medium Sand $\left(d_{50}=0.38 \mathrm{~mm}, \gamma^{\prime}=9.3 \mathrm{~m} \times 10^{3} \mathrm{~N} / \mathrm{m}^{3}\right.$, $\left.D_{r}=0.4\right)$

\begin{tabular}{|c|c|c|c|c|c|}
\hline $\begin{array}{l}\text { Test } \\
\text { number }\end{array}$ & $\begin{array}{c}D \\
(\mathrm{~m})\end{array}$ & $\begin{array}{c}W_{s} \\
(\mathrm{~N} / \mathrm{m})\end{array}$ & $\begin{array}{c}U \\
(\mathrm{~m} / \mathrm{s})\end{array}$ & $G$ & $F$ \\
\hline 1 & 0.050 & 1.358 & 0.084 & 0.058 & 0.120 \\
\hline 2 & 0.050 & 3.326 & 0.122 & 0.143 & 0.174 \\
\hline 3 & 0.050 & 4.996 & 0.130 & 0.215 & 0.186 \\
\hline 4 & 0.050 & 7.096 & 0.179 & 0.305 & 0.256 \\
\hline 5 & 0.050 & 5.898 & 0.158 & 0.254 & 0.226 \\
\hline 6 & 0.050 & 8.041 & 0.177 & 0.346 & 0.253 \\
\hline 7 & 0.050 & 8.041 & 0.184 & 0.346 & 0.263 \\
\hline 8 & 0.050 & 9.157 & 0.191 & 0.394 & 0.273 \\
\hline 9 & 0.050 & 10.143 & 0.210 & 0.436 & 0.300 \\
\hline 10 & 0.040 & 1.071 & 0.074 & 0.072 & 0.118 \\
\hline 11 & 0.040 & 2.034 & 0.077 & 0.137 & 0.123 \\
\hline 12 & 0.040 & 2.517 & 0.083 & 0.169 & 0.133 \\
\hline 13 & 0.040 & 3.060 & 0.123 & 0.206 & 0.196 \\
\hline 14 & 0.040 & 4.152 & 0.119 & 0.279 & 0.191 \\
\hline 15 & 0.040 & 4.873 & 0.148 & 0.328 & 0.237 \\
\hline 16 & 0.040 & 5.604 & 0.159 & 0.377 & 0.254 \\
\hline 17 & 0.040 & 6.324 & 0.174 & 0.425 & 0.279 \\
\hline 18 & 0.040 & 7.046 & 0.178 & 0.474 & 0.285 \\
\hline 19 & 0.040 & 7.046 & 0.181 & 0.474 & 0.289 \\
\hline 20 & 0.040 & 7.767 & 0.193 & 0.522 & 0.309 \\
\hline 21 & 0.040 & 8.477 & 0.214 & 0.570 & 0.342 \\
\hline 22 & 0.032 & 1.418 & 0.104 & 0.148 & 0.185 \\
\hline 23 & 0.032 & 2.517 & 0.131 & 0.227 & 0.234 \\
\hline 24 & 0.032 & 2.878 & 0.128 & 0.302 & 0.229 \\
\hline 25 & 0.032 & 2.878 & 0.142 & 0.302 & 0.254 \\
\hline 26 & 0.032 & 3.555 & 0.152 & 0.373 & 0.272 \\
\hline 27 & 0.032 & 1.418 & 0.102 & 0.149 & 0.183 \\
\hline
\end{tabular}

Scale effects should be investigated when the results of small-scale current-pipe-soil interaction tests are extrapolated to a real-life situation. The method of "modeling of models" was employed for investigating the scale effects in the current-pipesoil interaction tests. In our tests, the scale effects are examined by running three scales of test pipe diameter, i.e., $D=0.032$, 0.040 , and $0.050 \mathrm{~m}$, respectively (see Fig. 6). In the fields, the diameter of the commonly used pipelines is about $0.2-1.0 \mathrm{~m}$. That is, the pipeline was modeled at approximate 1/10 scale. As shown in Fig. 6, the results of test pipes with various diameters lie close to the same linear fitting line, when using Froude number $(\mathrm{F})$ and dimensionless pipeline submerged weight $(G)$ for data correlation analysis. This indicates that the scale effects are not obvious for the range of pipeline diameters examined and within the examined Froude range, i.e., $0.10<F<0.35$. Nevertheless, the test results need further validation with future fullscale experiments.

The effect of soil particle size is also important for the currentpipe-soil interaction problem. The properties of the seabed may vary at different ocean zones, thus it is worthy of examining the influence of soil properties upon the pipeline lateral stability. Besides the aforementioned tests on medium sand, an additional seven series of experiments have been conducted on a fine-sand seabed, whose properties are given in Table 3. The test results for the fine sand are listed in Table 5.

A comparison of F- $G$ correlations for pipeline lateral stability on medium sand with that on fine sand is given in Fig. 7.

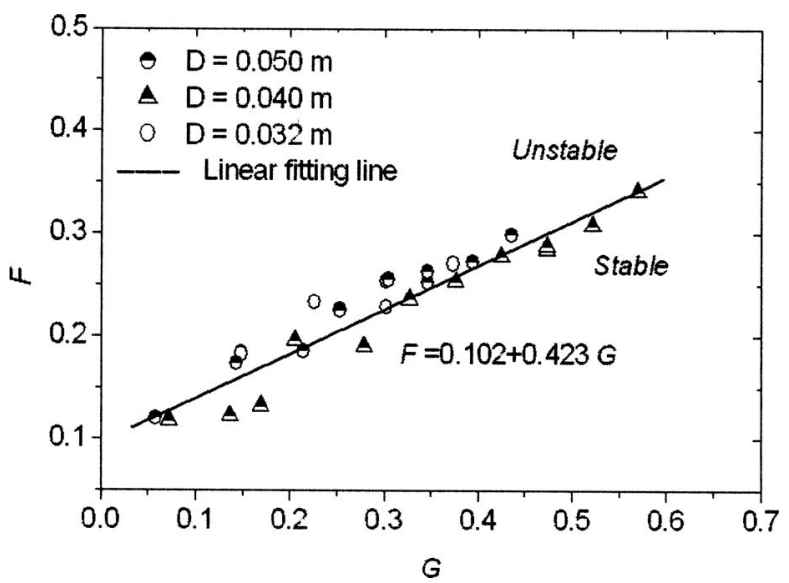

Fig. 6. F- $G$ correlation for the lateral stability of pipes with various diameters (medium sands)

As shown in the figure, there exists another linear relationship between Froude number and the dimensionless submerged weight of the pipeline within the examined Froude range $(0.10<\mathrm{F}<0.25)$

$$
\mathrm{F}=0.101+0.205 \mathrm{G} \quad \text { (for a fine-sand seabed) }
$$

The comparison of F- $G$ correlations for the two kinds of sands indicates that the pipelines on the medium-sand seabed are more stable than on the fine-sand one (see Fig. 5). For the two kinds of sands, both of them are of medium density $\left(1 / 3<D_{r}<2 / 3\right)$; therefore the main difference is in particle size. According to Shields parameter (see Sumer and Fredsoe 2002), the fine sand is easier to be scoured than the medium sand for the same conditions for flow and pipeline. The sand scour around the pipeline, especially behind the pipeline, would reduce the passive pressure upon the pipeline, which would result in the pipeline losing lateral stability easier. However, with the decrease of submerged weight of the pipeline, sand particle size effects on the pipeline instability (in terms of Froude number) decrease, as shown in Fig. 7.

\section{Comparison with Pipeline Stability in Waves}

As previously mentioned, pipeline stability in waves has been investigated by many researchers. It would be interesting and worth making a comparison between pipeline stability in currents and that in waves.

Table 5. Experimental Results of Currents-Induced Lateral Stability of Pipeline on Fine Sand $\left(d_{50}=0.12 \mathrm{~mm}, \gamma^{\prime}=8.7 \mathrm{~m} \times 103 \mathrm{~N} / \mathrm{m}^{3}, D_{r}=0.6\right)$

\begin{tabular}{lccccc}
\hline $\begin{array}{l}\text { Test } \\
\text { number }\end{array}$ & $\begin{array}{c}D \\
(\mathrm{~m})\end{array}$ & $\begin{array}{c}W_{s} \\
(\mathrm{~N} / \mathrm{m})\end{array}$ & $\begin{array}{c}U \\
(\mathrm{~m} / \mathrm{s})\end{array}$ & $G$ & $\mathrm{~F}$ \\
\hline 28 & 0.050 & 1.735 & 0.082 & 0.079 & 0.117 \\
29 & 0.050 & 3.761 & 0.097 & 0.172 & 0.139 \\
30 & 0.050 & 5.438 & 0.104 & 0.249 & 0.148 \\
31 & 0.050 & 6.345 & 0.113 & 0.290 & 0.161 \\
32 & 0.050 & 7.344 & 0.121 & 0.336 & 0.173 \\
33 & 0.050 & 9.429 & 0.129 & 0.431 & 0.185 \\
34 & 0.050 & 11.427 & 0.148 & 0.522 & 0.212 \\
\hline
\end{tabular}




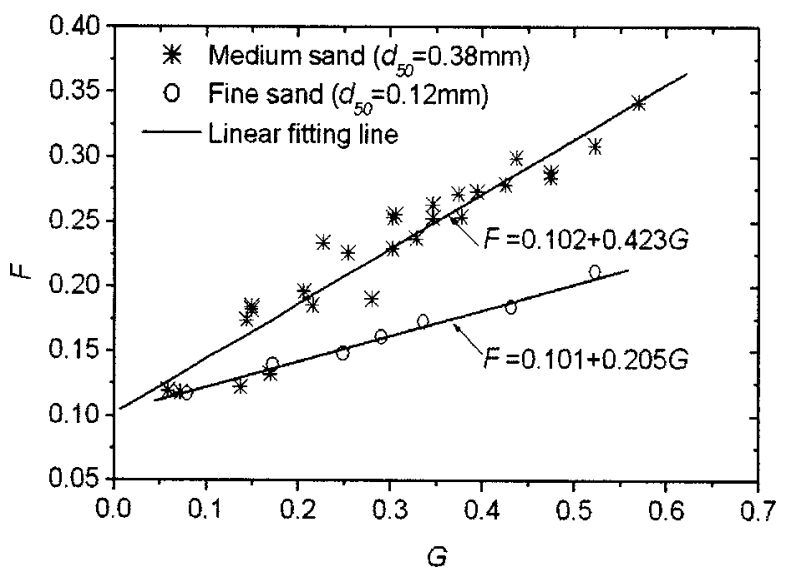

Fig. 7. Effects of sand particle size on pipeline lateral stability in currents

For the wave-induced pipeline lateral stability, the KeuleganCarpenter number should be further taken into account, which is defined as

$$
\mathrm{KC}=\frac{U_{m} T}{D}
$$

where $U_{m}=$ maximum value of wave-induced water particle velocity; and $T=$ wave period. The Froude number for the pipeline in waves is defined as

$$
\mathrm{F}=\frac{U_{m}}{\sqrt{g D}}
$$

In general, the $\mathrm{KC}$ number controls the generation and development of the vortex around the pipeline in waves, and is related to the hydrodynamic force on the pipeline. In the physical modeling of wave-induced pipeline stability by Gao et al. (2003), 18 series of tests on a kind of medium sand seabed have been conducted in a U-shaped oscillatory flow tunnel. In those experiments, the range of $\mathrm{KC}$ is from 5 to 20 , which covers the typical environmental conditions in the South China Sea. The test soil was a kind of medium sand, whose physical properties are the same as those of the medium sand in this study (see Table 3).

The physical phenomena of pipeline losing lateral stability in currents is distinguished greatly from those in waves. The typical processes of the pipeline losing lateral stability in waves and that in currents are illustrated in Fig. 8. Under wave loading, after the totally stable stage, the slight rocking of pipeline is followed, whose period is approximately the same as the wave period. After the duration of slight rocking, the pipeline suddenly moves back and forth with large horizontal displacements, i.e., the pipe loses lateral stability [see Fig. 8(a)]. However, under the loading of currents, the pipe pushes the soil nearby ahead with a slight lateral displacement in the stream direction instead of with slight rocking, after the totally stable stage. In the stage of the pipeline losing stability, the pipeline in the currents moves with large displacements only in the stream direction [see Fig. 8(b)].

For the case of ocean currents $(T \gg 1 \mathrm{~s})$, the $\mathrm{KC}$ number can be regarded as infinity, i.e., $\mathrm{KC}=\infty$. In currents, the hydrodynamic loads upon the pipeline are mainly the drag forces due to flow velocity. However, in waves, the hydrodynamic loads include not only the drag forces but also the inertial forces due to the acceleration of the water particle. A comparison of experimental data
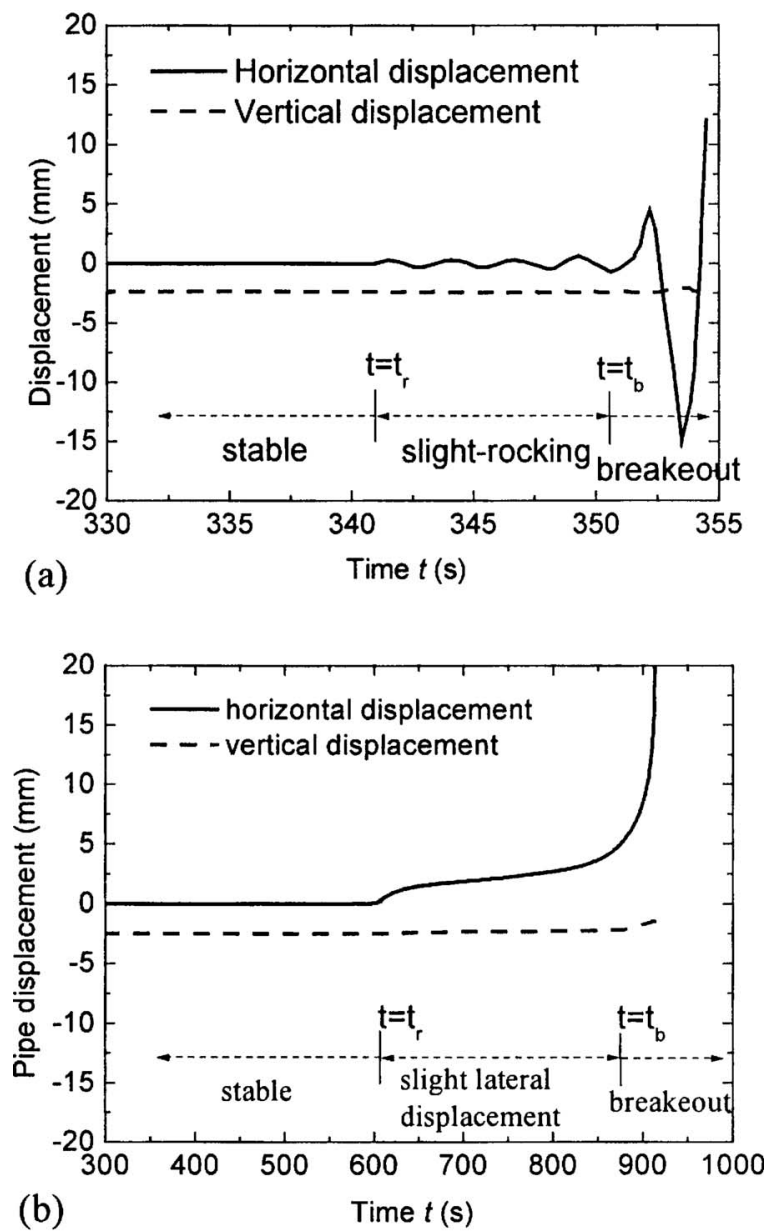

Fig. 8. Typical pipe displacement-time curves: (a) in waves; (b) in currents

for pipeline lateral stability in currents and those in waves is shown in Fig. 9. The figure indicates there exist different linear relationships for pipeline lateral stability in waves and in currents. In the same soil conditions (e.g, a medium sand seabed), for a given value of nondimensional pipeline submerged weight $(G)$, the Froude number $(F)$ inducing pipeline instability in currents is

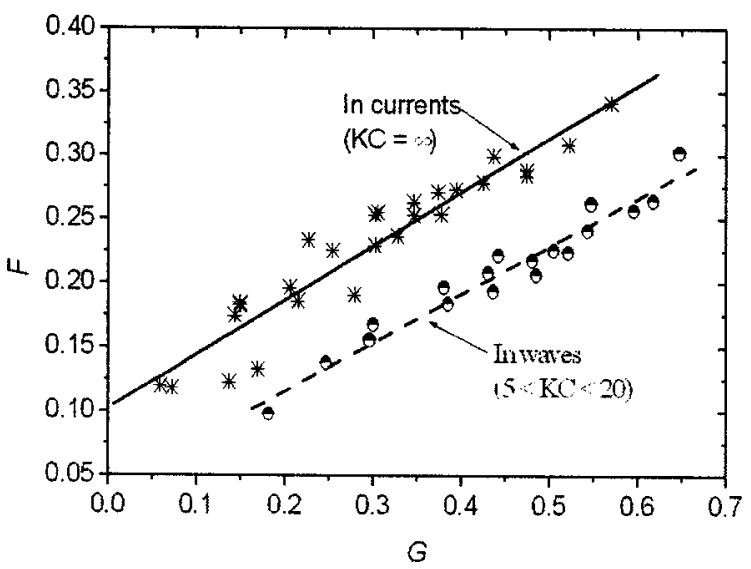

Fig. 9. Comparison between pipeline lateral stability in currents and that in waves (medium sands) 
larger than in waves. This indicates that the pipeline directly laid on the sandy seabed in currents remains more stable than in waves for the same level of flow velocity.

\section{Concluding Remarks}

According to the similarity analysis, ocean currents induced lateral stability of pipeline on the sandy seabed was simulated in a flow flume. Based on the results of a total of 34 series of tests, the following conclusions can be drawn: (1) there usually exists three characteristic times in the process of pipeline losing lateral stability: onset of sand scour; slight lateral displacement of pipeline; and breakout of pipeline; (2) a linear relationship between Froude number and the dimensionless submerged weight of the pipeline is established within the examined Froude range $(0.10<F<0.35)$ for describing the stability of pipelines laid directly upon a sandy seabed; and (3) the influence of sand particle size on pipeline lateral stability becomes much more obvious with the increase of submerged weights of pipeline or at higher values of Froude number.

A comparison has been made between pipeline stability in currents and in waves. The physical phenomena of the pipeline losing lateral stability in currents are somewhat different from those in waves, especially in the stage of pipe breakout. For the same level of flow velocity, the pipeline laid directly on the sandy seabed in currents remains more stable than in waves. Note that scale effects should be carefully examined when extrapolating the results of small-scale current-pipe-soil interaction experiments to real-life situations.

\section{Acknowledgments}

Financial support by National Natural Science Foundation of China (Grant Nos. 50509022 and 10532070) and "Eleventh FiveYear Plan" of the Chinese Academy of Sciences (Grant No. KJCX2-YW-L02) is greatly appreciated.

\section{References}

Allen, D. W., Lammert, W. F., Hale, J. R., and Jacobsen, V. (1989). "Submarine pipeline on-bottom stability: recent AGA research."
Proc., 21st Annual Offshore Technology Conf., Houston, OTC 6055, 121-132.

Brennodden, H., Lieng, J. T., Sotberg, T., and Verley, R. L. P. (1989). "An energy-based pipe-soil interaction model." Proc., 21st Annual Offshore Technology Conf., Houston, OTC 6057, 147-158.

Brennodden, H., Sveggen, O., Wagner, D. A., and Murff, J. D. (1986). "Full-scale pipe-soil interaction tests." Proc., 18th Annual Offshore Technology Conf., Houston, OTC 5338, 433-440.

Chakrabarti, K. (1994). Offshore structure modeling, JBW Printers and Binder Pte. Ltd., Singapore.

Det Norske Veritas. (1988). On-bottom stability design of submarine pipeline, Recommended Practice E305, Hovik, Norway.

Gao, F. P., Gu, X. Y., and Jeng, D. S. (2003). "Physical modeling of untrenched submarine pipeline instability." Ocean Eng., 30, 1283-1304.

Gao, F. P., Gu, X. Y., Jeng, D. S., and Teo, H.T. (2002). “An experimental study for wave-induced instability of pipelines: The breakout of pipelines." Appl. Ocean. Res., 24, 83-90.

Gao, F. Jeng, D.-S., and Wu, Y. (2006). "Improved analysis method for wave-induced pipeline stability on sandy seabed." J. Transp. Eng., 132(7) 590-596.

Jones, W. T. (1978). "On-bottom pipeline stability in steady water currents." JPT, J. Pet. Technol., 30, 475-484.

Lawlor, C. D. F., and Flynn, S. J. A. (1991). "Subsea pipeline stability analysis: still a black art?" Trans., Institution of Engineers, Australian Civil Engineering, 1, 1-8.

Palmer, A. C., Steenfelt, J. S., and Jacobsen, V. (1988). "Lateral resistance of marine pipelines on sand." Proc., 20th Annual Offshore Technology Conf., OTC 5853, 399-408.

Sumer, B. M., and Fredsoe, J. (2002). The mechanics of scour in the marine environment, World Scientific, River Edge, N.J.

Taylor, R. N. (1995). Geotechnical centrifuge technology, Blackie Academic and Professional, Chapman and Hall, London.

Teh, T. C., Palmer, A. C., and Damgaard, J. S. (2003). "Experimental study of marine pipelines on unstable and liquefied seabed." Coastal Eng., 50, 1-17.

Wagner, D. A., Murff, J. D., Brennodden, H., and Sveggen, O. (1987). "Pipe-soil interaction model." Proc., 19th Annual Offshore Technology Conf., Houston, OTC 5504, 181-190.

Zhang, J, Stewart, D. P., and Randolph, M. F. (2002). "Modeling of shallowly embedded offshore pipelines in calcareous sand." J. Geotech. Geoenviron. Eng., 128(5), 363-371. 